\title{
GROWTH AND YIELD OF SHALLOT UNDER SEVERAL LEVELS OF SOIL WATER TABLE
}

\author{
Susilawati ${ }^{\star}$, Irmawati, Sukarmi Sri, Ammar Muhammad, \\ Kurnianingsih Astuti, Yusnita, Yayandra \\ Department of Agronomy, Faculty of Agriculture, University of Sriwijaya, Indonesia \\ *E-mail: susilawati@fp.unsri.ac.id
}

\begin{abstract}
The research was aimed to evaluate differences on growth and yield of two shallot varieties under several levels of soil water table. It was conducted in experimental field and plastic house of Agriculture Faculty, Universitas Sriwijaya for three months in 2019. Materials used were shallot bulb from Tajuk and Pancasona varieties and biochar originated from oil palm empty fruit bunch. Factorial Randomized Block Design was used with two treatment factors and three replicates. First treatment was 2 shallot variety consisting Tajuk and Pancasona variety. Second treatment was level of soil water table including $T_{0}=0 \mathrm{~cm}$ below soil surface; $T_{1}=10 \mathrm{~cm}$ below soil surface; $T_{2}=15 \mathrm{~cm}$ below soil surface; and $T_{3}=20 \mathrm{~cm}$ below soil surface. The observed parameters consisted of plant height, leaf number, tiller number, total bulb fresh weight, bulb fresh weight, bulb dry weight, bulb number, bulb diameter, and root length. Results showed that shallot growth of Pancasona and Tajuk variety was highly affected by levels of soil water table. It was then concluded that Pancasona variety showed better growth performance and yield compared to Tajuk variety. The deeper soil water table reaching $20 \mathrm{~cm}$ below soil surface can reduce growth and yield of shallot.
\end{abstract}

\section{KEY WORDS}

Non-tidal swamp, shallot variety, water table.

Indonesia is one of seasonal vegetables and fruits producer. Form total 22 types of seasonal vegetables, five main commodities with highest production in 2017 were shallot, cabbage, cayenne pepper, potato, and chili pepper. Shallot production reached 1.47 million tons from total 12.48 million tons or approximately $11.78 \%$ (Statistics Indonesia, 2017; Agricultural Statistics, 2018). Therefore, shallot has been an important commodity for national economy development.

Shallot production centre areas in Indonesia include Central Java, East Java, West Java and West Nusa Tenggara. These four provinces have contributed about $85.33 \%$ of Indonesian average production. East Java shares the biggest contribution of $40.59 \%$ with 432,813 tons of average production. While East Java in the second place with $23.16 \%$ and 246,927 tons of average production per year. West Java and West Nusa Tenggara contribute $11.10 \%$ and $10.48 \%$, respectively. The rest of $14.67 \%$ is from other provinces (Agriculture Data and Information Center, 2016).

Based on General Directorate of Horticulture, the success key of shallot self-sufficiency is on the management and development of production centers that are not solely concentrated in Java. At this moment, shallot cultivation in large scale is not only found in Brebes, Cirebon or Nganjuk, but also has been spread in Solok, Bali, Bima, Sumbawa, Belu, Malaka, Southeast Maluku, Enrekang, Tapin and other areas. West Sumatra province, especially Solok, is shallot production center in Sumatra with total production reaching 955,000 tons in 2017. While shallot domestic demand in West Sumatra is about $2.57 \mathrm{~kg} / \mathrm{year}$ or about 14,000 tons, the production surplus is then distributed to North Sumatra, South Sumatra, Riau and Jambi (Sudarsono, 2018). Shallot production in South Sumatra in 2015, 2016 and 2017 is around 582.8 tons, 637.6 tons and 1,375.8 tons, respectively (South Sumatra Statistics Center, 2018).

Along with government program of shallot production center development outside Java, South Sumatra is considered to be potential for the option. South Sumatra has the largest 
coverage areas of non-tidal swamp in Sumatra reaching 2.98 millions ha. From the total, there are about 368,690 ha of non-tidal swamp area cultivated as rice field, consisted of 70,908 ha of shallow swamp, 129,103 ha of middle swamp, and 168,670 ha of deep swamp. While the rest for about 2.60 millions ha are not yet utilized. In 2019, government optimization program of 500,000 ha swamp -both non-tidal and tidal swamp-includes South Sumatra, along with other five provinces which are South Kalimantan, South Sulawesi, Lampung, Jambi and Central Kalimantan. South Sumatra and South Kalimantan are considered as the most ready provinces (Rajagukguk, 2019).

Up until now, non-tidal swamp development is still not optimal due to its flooding problem with various water depths and flooding durations. Flooding have a great impact on environment and yield (Jackson and Ismail, 2015; Malik et al., 2015). Main challenge for nontidal swamp cultivation is how to optimize seasonal water availability. The land can be flooded for several months during rainy season so that it can be utilized for cultivation and also is suffered from drought in dry season (Meihana et al., 2019a). Anoxia condition occurs during flooding, while hypoxia occurs when water depth is less than $20 \mathrm{~cm}$ (Malik et al., 2015; Meihana et al., 2019b).

Shallot growth demands a growing media containing crumble soil structure and sufficient organic matter with non-acid soil pH for about 5.6-6.5 (Sumarni and Hidayat, 2005; Kurnianingsih et al., 2018a; Kurnianingsih et al., 2018b). Generally, shallot will not have optimal growth when being cultivated in rainy season. Main factor for the problem is water availability. Susilawati et al. (2018) reported that the combination of $60 \%$ of soil and $40 \%$ cow manure for growing media resulted in the best shallot growth and yield with average of 84.36 $\mathrm{g}$ of dry weight bulb per clump. In contrast, shallot cultivation during dry season will face water shortage problem. However, it is necessary to evaluate how deep soil water table in order to be able to support shallot growth. Research study by Susilawati and Lakitan (2019) resulted that the best performing snap bean growth and yield was obtained in $20 \mathrm{~cm}$ of water depth below soil surface. Thus, this research then was conducted to evaluate the difference of growth ability and yield of two shallot varieties under several levels of soil water table cultivated in polybags.

\section{METHODS OF RESEARCH}

The research was conducted in experimental farm and in plastic house of Agriculture Faculty, Universitas Sriwijaya Indralaya in Ogan Ilir, South Sumatra. While laboratory works were carried out in Laboratory of Plant Physiology, Agronomy Department, Universitas Sriwijaya Indralaya. The research was conducted for three months in 2019. Materials used consisted of shallot bulbs from Tajuk and Pancasona varieties, biochar from oil palm empty fruit bunch, paper bag and polybag. Research tools consisted of hoe, gauge, plastic buckets, measuring glass, analytical scale and oven. Factorial Randomized Block Design was used with two factors and three replicates. First factor was shallot variety: $\mathrm{V}_{1}=$ Tajuk variety; and $\mathrm{V}_{2}=$ Pancasona variety. Second factor was soil water table: $\mathrm{T}_{0}=0 \mathrm{~cm}$ below soil surface; $T_{1}$ $=10 \mathrm{~cm}$ below soil surface; $T_{2}=15 \mathrm{~cm}$ below soil surface; and $T_{3}=20 \mathrm{~cm}$ below soil surface.

Planting media was alluvial top soil cleaned and sieved before putting into the polybags of $10 \mathrm{~kg}$ volume. Biochar then was mixed into the media one week before planting with the dosage of 20 tons per ha. Shallot bulbs from Tajuk and Pancasona variety were obtained from Brebes. Before planted, the bulbs were cut in 1/3 from the edge and then planted into the media in $2-3 \mathrm{~cm}$ depth. One bulb was planted into each polybag. The polybags containing shallot bulbs then were soaked into plastic bucket filled with water according to the water table treatments and then put in the plastic house.

Water table in the bucket was maintained as the treatments by adding water every evening. Shallot plant treated with water table treatment only survived for 11 days, so the polybags then were taken out of the bucket in the $12^{\text {th }}$ day. After taken out, polybags were still placed in the plastic house until 30 days. Polybags then were placed outside plastic house in $31^{\text {th }}$ day. Parameters observed consisted of plant height, leaf number, and tiller number which were all measured from $3-38$ days after treatment (DAT) and total plant fresh 
weight, bulb fresh weight, bulb dry weight, bulb number, bulb diameter, root length and added water volume.

Resulted data then were statistically analyzed using analysis of variance (ANOVA) by comparing the resulted $F$ value to $F$ table. When resulted $F$ value less than $F$ table at $5 \%$, it means that the treatment has insignificant effect. While significant effect is obtained if $F$ value is bigger than $\mathrm{F}$ table at $5 \%$ level. Furthermore, very significant effect is obtained if resulted $\mathrm{F}$ value is more than $\mathrm{F}$ table at $1 \%$ level. Least significant difference test then used to analyze the data that showed significant and very significant effect.

Table 1 - The effect of soil water table on vegetative growth of two shallot varieties

\begin{tabular}{|c|c|c|c|c|c|}
\hline \multirow{2}{*}{ No. } & \multirow{2}{*}{ Parameter } & \multicolumn{3}{|l|}{ F-value } & \multirow{2}{*}{ Coefficient of variance (\%) } \\
\hline & & $\mathrm{V}$ & $\mathrm{T}$ & VT & \\
\hline 1. & Plant height at treatment or 0 DAT & $0.19^{\mathrm{ns}}$ & $2.62^{\text {ns }}$ & $1.99^{\mathrm{ns}}$ & 22.14 \\
\hline 2. & Plant height at 3 DAT & $2.17^{\mathrm{ns}}$ & $3.14^{\mathrm{ns}}$ & $3.58^{*}$ & 11.41 \\
\hline 3. & Plant height at 10 DAT & $1.35^{\mathrm{ns}}$ & $2.35^{\mathrm{ns}}$ & $0.97^{\mathrm{ns}}$ & 13.65 \\
\hline 4. & Plant height at 17 DAT & $2.73^{\mathrm{ns}}$ & $2.62^{\mathrm{ns}}$ & $2.77^{\mathrm{ns}}$ & 10.20 \\
\hline 5. & Plant height at 24 DAT & $4.17^{\mathrm{ns}}$ & $4.05^{*}$ & $3.50^{*}$ & 10.31 \\
\hline 6. & Plant height at 31 DAT & $33.79^{\star x}$ & $1.62^{\mathrm{ns}}$ & $4.27^{*}$ & 8.58 \\
\hline 7. & Leaf number at treatment or 0 DAT & $0.68^{\mathrm{ns}}$ & $1.11^{\mathrm{ns}}$ & $0.05^{\mathrm{ns}}$ & 31.56 \\
\hline 8. & Leaf number at 3 DAT & $0.31^{\mathrm{ns}}$ & $2.00^{\mathrm{ns}}$ & $0.26^{\text {ns }}$ & 28.01 \\
\hline 9. & Leaf number at $10 \mathrm{DAT}$ & $1.00^{\mathrm{ns}}$ & $2.36^{\mathrm{ns}}$ & $0.35^{\mathrm{ns}}$ & 27.68 \\
\hline 10. & Leaf number at $17 \mathrm{DAT}$ & $3.20^{\mathrm{ns}}$ & $2.29^{\mathrm{ns}}$ & $0.30^{\mathrm{ns}}$ & 27.58 \\
\hline 11. & Leaf number at 24 DAT & $11.25^{\pi x}$ & $2.34^{\mathrm{ns}}$ & $0.82^{\mathrm{ns}}$ & 28.13 \\
\hline 12. & Leaf number at 31 DAT & $9.57^{\star *}$ & $1.67^{\mathrm{ns}}$ & $0.60^{\mathrm{ns}}$ & 32.40 \\
\hline 13. & Tiller number at treatment or 0 DAT & $5.22^{*}$ & $1.06^{\mathrm{ns}}$ & $1.36^{\mathrm{ns}}$ & 22.26 \\
\hline 14. & Tiller number at 3 DAT & $12.00^{\pi x}$ & $3.20^{\mathrm{ns}}$ & $7.75^{\star \pi}$ & 14.97 \\
\hline 16. & Tiller number at $10 \mathrm{DAT}$ & $8.23^{*}$ & $2.57^{\mathrm{ns}}$ & $3.48^{*}$ & 17.53 \\
\hline 17. & Tiller number at $17 \mathrm{DAT}$ & $4.77^{\star}$ & $0.68^{\mathrm{ns}}$ & 3.36 * & 20.18 \\
\hline 18. & Tiller number at $24 \mathrm{DAT}$ & $5.38^{*}$ & $1.52^{\mathrm{ns}}$ & $2.44^{\mathrm{ns}}$ & 17.35 \\
\hline 19. & Tiller number at $31 \mathrm{DAT}$ & $1.10^{\mathrm{ns}}$ & $2.41^{\mathrm{ns}}$ & $0.62^{\mathrm{ns}}$ & 26.62 \\
\hline \multirow[t]{3}{*}{20.} & Root length & $16.67^{* *}$ & $4.73^{*}$ & $1.14^{\mathrm{ns}}$ & 21.11 \\
\hline & $\mathrm{F}$ table $5 \%$ & 4.60 & 3.34 & 3.34 & \\
\hline & F table $1 \%$ & 8.86 & 5.56 & 5.56 & \\
\hline
\end{tabular}

Note: $D A T=$ day after treatment; ns = not significantly different; * = significantly different at $p<0.05 ;$ and ${ }^{* *}=$ significantly different at $p<0.01$.

\section{RESULTS OF STUDY}

Based on analysis of variance, it was resulted that levels of soil water table had significant effect on growth and yield of two shallot varieties as seen in Table 1. Difference on varieties showed very significant effect on plant height at 24 DAT and 31 DAT, tiller number at $0-24$ DAT, and root length, while soil water table treatment only showed significant effect at 24 DAT. The combination of variety and soil water table treatment had insignificant effect on all observed parameters, except for plant height at 3, 24, and 31 DAT, and also for tiller number at $3-17$ DAT.

The difference on soil water table showed insignificant effect on growth of two shallot varieties for 11 days after planting, as seen in several parameters such as plant height, leaf number and tiller number at 0 DAT. Based on average data, $V_{1}$ (Tajuk) had shorter height compared to $V_{2}$ (Pancasona) from 0 to 24 DAT, the difference between these two varieties were significantly showed at 31 DAT. Water table treatment showed insignificant effect on plant height at all plant ages, except at 24 DAT. The difference on leaf number parameter was started to appear from 0 to 17 DAT but was statistically significant at 24 and 31 DAT. Leaf number was not affected by soil water table treatment (Table 2).

Similar with plant height and leaf number, Pancasona also showed better result in tiller number compared to Tajuk variety. Besides, soil water table treatment also did not show any effect on tiller number. However, tiller number of these two varieties statistically showed significant difference started from 0 to 24 DAT, except at 31 DAT. Root length of Tajuk and Pancasona was significantly difference, while soil water table at $0 \mathrm{~cm}$ also showed significant difference compared to other depth treatment (Table 3). 
Soil water table treatment showed very significant effect on growth of two shallot varieties as seen in the parameters of plant height at 3,24 and 31 DAT and tiller number at 3, 10 and 17 DAT. Least significant difference (LSD) test on $5 \%$ level showed on three different plant ages showed that the lowest plant height result was obtained from the same combination treatment which was $\mathrm{V}_{1} \mathrm{~T}_{3}$ with $14.52 \mathrm{~cm}$ at 3 DAT, $18.17 \mathrm{~cm}$ at 24 and 31 DAT. The same pattern was also resulted in tiller number where the lowest result was on $V_{1} T_{1}$ (Tajuk variety with $10 \mathrm{~cm}$ of water table below soil surface) with 2.5 at 3 DAT, 3.0 at 10 DAT and 3.33 at 17 DAT. The lowest result was all obtained from the same variety which was Tajuk variety (Table 4).

Table 2. Plant height of two shallot varieties due to soil water table treatment

\begin{tabular}{|c|c|c|c|c|c|c|}
\hline \multirow[t]{3}{*}{ Treatment } & \multicolumn{6}{|c|}{ Plant age (DAT $=$ days after treatment) } \\
\hline & 0 & 3 & 10 & 17 & 24 & 31 \\
\hline & \multicolumn{6}{|c|}{ Plant height $(\mathrm{cm})$} \\
\hline \multicolumn{7}{|l|}{ Variety } \\
\hline $\mathrm{V}_{1}$ & $11.18 \pm 3.91 a$ & $17.28 \pm 6.85 a$ & $20.47 \pm 8.90 a$ & $23.18 \pm 9.94 a$ & $24.22 \pm .12 .40 \mathrm{a}$ & $22.26 \pm .8 .54 a$ \\
\hline $\mathrm{V}_{2}$ & $11.63 \pm 8.54 a$ & $18.51 \pm 6.11 \mathrm{a}$ & $21.84 \pm 2.01 \mathrm{a}$ & $25.51 \pm 1.96 a$ & $26.40 \pm 1.03 a$ & $27.31 \pm 2.67 \mathrm{~b}$ \\
\hline LSD & & & & & & 1.86 \\
\hline \multicolumn{7}{|c|}{ Soil water table $(\mathrm{cm})$} \\
\hline 0 & $9.49 \pm 7.39 a$ & $18.19 \pm 7.18 a$ & $23.17 \pm 3.18 a$ & $26.64 \pm .2 .37 a$ & $27.10 \pm 1.70 b$ & $26.01 \pm 5.98 a$ \\
\hline 10 & $12.43 \pm 0.00 a$ & $17.46 \pm 3.71 \mathrm{a}$ & $20.61 \pm 4.49 a$ & $24.65 \pm .3 .96 a$ & $26.10 \pm 2.40 a$ & $25.04 \pm 5.83 a$ \\
\hline 15 & $13.12 \pm 6.15 a$ & $19.74 \pm 6.75 a$ & $21.91 \pm 1.73 a$ & $24.79 \pm .0 .88 a$ & $25.83 \pm 0.71 \mathrm{a}$ & $24.77 \pm 9.12 a$ \\
\hline 20 & $10.56 \pm 5.06 a$ & $16.20 \pm 7.14 \mathrm{a}$ & $18.94 \pm 8.59 a$ & $22.57 \pm .13 .72 \mathrm{a}$ & $22.19 \pm 17.08 a$ & $23.33 \pm 21.92 a$ \\
\hline \multirow[t]{2}{*}{ LSD } & & & & & 4.57 & \\
\hline & \multicolumn{6}{|l|}{ Leaf number } \\
\hline$\overline{V_{1}}$ & $7.42 \pm 3.18 a$ & $10.44 \pm 4.04 a$ & $12.46 \pm 6.14 a$ & $13.00 \pm .6 .34 a$ & $11.00 \pm .6 .54 a$ & $11.33 \pm .5 .43 a$ \\
\hline \multirow[t]{2}{*}{$\mathrm{V}_{2}$} & $8.25 \pm 3.38 a$ & $11.13 \pm 6.76 a$ & $13.96 \pm 8.45 a$ & $15.92 \pm 9.15 a$ & $16.25 \pm 9.85 a$ & $17.17 \pm 10.78 b$ \\
\hline & & & & & 4.74 & 5.71 \\
\hline \multicolumn{7}{|c|}{ Soil water table (cm) } \\
\hline 0 & $8.58 \pm 3.18 a$ & $12.75 \pm 5.30 a$ & $16.08 \pm .8 .13 a$ & $17.75 \pm 9.55 \mathrm{a}$ & $17.00 \pm 13.44 a$ & $17.67 \pm 16.26 a$ \\
\hline 10 & $8.25 \pm 1.06 a$ & $9.25 \pm 1.06 a$ & $12.17 \pm .1 .41 \mathrm{a}$ & $13.33 \pm 0.71 \mathrm{a}$ & $11.83 \pm 2.12 \mathrm{a}$ & $12.58 \pm 4.60 \mathrm{a}$ \\
\hline 15 & $8.25 \pm 1.06 a$ & $11.75 \pm 0.35 a$ & $13.83 \pm .0 .71 \mathrm{a}$ & $14.75 \pm 7.42 \mathrm{a}$ & $13.67 \pm 14.85 a$ & $14.33 \pm 18.38 a$ \\
\hline 20 & $6.25 \pm 1.77 a$ & $9.38 \pm 0.88 a$ & $10.75 \pm .3 .89 a$ & $12.00 \pm 7.07 \mathrm{a}$ & $12.00 \pm 14.14 a$ & $12.42 \pm 10.25 a$ \\
\hline
\end{tabular}

The numbers followed by same letter in the same row means insignificant difference.

Table 3 - Tiller number and root length of two shallot varieties due to soil water table treatment

\begin{tabular}{|c|c|c|c|c|c|c|}
\hline \multirow[t]{2}{*}{ Treatment } & \multicolumn{6}{|c|}{ Plant age (DAT = days after treatment) } \\
\hline & 0 & 3 & 10 & 17 & 24 & 31 \\
\hline & \multicolumn{6}{|l|}{ Tiller number } \\
\hline \multicolumn{7}{|l|}{ Variety } \\
\hline $\mathrm{V}_{1}$ & $2.88 \pm 1.25 a$ & $3.17 \pm 1.96 a$ & $3.46 \pm 1.25 a$ & $4.00 \pm .1 .68 \mathrm{a}$ & $4.42 \pm .1 .55 a$ & $5.50 \pm .2 .04 a$ \\
\hline $\mathrm{V}_{2}$ & $3.54 \pm 1.44 b$ & $3.92 \pm 2.33 b$ & $4.25 \pm 2.60 \mathrm{~b}$ & $4.79 \pm 2.59 \mathrm{~b}$ & $5.21 \pm 2.43 b$ & $6.17 \pm 4.22 \mathrm{a}$ \\
\hline LSD & 0.51 & 0.37 & 0.48 & 0.63 & 0.59 & \\
\hline \multicolumn{7}{|c|}{ Soil water table $(\mathrm{cm})$} \\
\hline 0 & $3.50 \pm 2.83 a$ & $3.92 \pm .4 .60 a$ & $4.42 \pm 4.60 \mathrm{a}$ & $4.83 \pm 4.24 \mathrm{a}$ & $5.33 \pm 2.83 \mathrm{a}$ & $7.25 \pm 3.18 \mathrm{a}$ \\
\hline 10 & $2.92 \pm 2.47 a$ & $3.17 \pm .2 .83 a$ & $3.50 \pm 2.12 \mathrm{a}$ & $4.25 \pm 3.89 \mathrm{a}$ & $4.67 \pm 4.24 \mathrm{a}$ & $5.75 \pm 3.18 \mathrm{a}$ \\
\hline 15 & $3.42 \pm 0.35 a$ & $3.83 \pm .0 .71 \mathrm{a}$ & $4.00 \pm 0.00 \mathrm{a}$ & $4.17 \pm 0.00 \mathrm{a}$ & $4.92 \pm 0.35 \mathrm{a}$ & $5.25 \pm 0.35 \mathrm{a}$ \\
\hline 20 & $3.00 \pm 0.00 a$ & $3.25 \pm .0 .35 a$ & $3.50 \pm 0.00 \mathrm{a}$ & $4.33 \pm 1.41 \mathrm{a}$ & $4.33 \pm 0.71 \mathrm{a}$ & $5.08 \pm 1.06 \mathrm{a}$ \\
\hline & \multicolumn{6}{|c|}{ Root length (cm) } \\
\hline \multicolumn{7}{|l|}{ Variety } \\
\hline $\mathrm{V}_{1}$ & $\mathrm{~nm}$ & $\mathrm{~nm}$ & $\mathrm{~nm}$ & $\mathrm{~nm}$ & $\mathrm{~nm}$ & $8.73 \pm .2 .15 a$ \\
\hline $\mathrm{V}_{2}$ & $\mathrm{~nm}$ & $\mathrm{~nm}$ & $\mathrm{~nm}$ & $\mathrm{~nm}$ & $\mathrm{~nm}$ & $12.46 \pm 10.03 b$ \\
\hline LSD & & & & & & 1.95 \\
\hline \multicolumn{7}{|c|}{ Soil water table $(\mathrm{cm})$} \\
\hline 0 & $\mathrm{~nm}$ & $\mathrm{~nm}$ & $\mathrm{~nm}$ & $\mathrm{~nm}$ & $\mathrm{~nm}$ & $13.50 \pm 16.26 \mathrm{~b}$ \\
\hline 10 & $\mathrm{~nm}$ & $\mathrm{~nm}$ & $\mathrm{~nm}$ & $\mathrm{~nm}$ & $\mathrm{~nm}$ & $9.00 \pm 3.54 \mathrm{a}$ \\
\hline 15 & $\mathrm{~nm}$ & $\mathrm{~nm}$ & $\mathrm{~nm}$ & $\mathrm{~nm}$ & $\mathrm{~nm}$ & $9.92 \pm 4.24 \mathrm{a}$ \\
\hline 20 & $\mathrm{~nm}$ & $\mathrm{~nm}$ & $\mathrm{~nm}$ & $\mathrm{~nm}$ & $\mathrm{~nm}$ & $9.96 \pm 7.60 \mathrm{a}$ \\
\hline LSD & & & & & & 2.39 \\
\hline
\end{tabular}

The numbers followed by same letter in the same row means insignificant difference; nm=not measure.

Shallot variety showed significant effect on yield component as obtained from the parameters of bulb fresh weight, bulb dry weight, bulb diameter and harvest index, while water table treatment had significant effect on total plant fresh weight (shoot + bulb) and bulb fresh weight. 
Table 4 - Combinations of variety and soil water table treatment on plant height and tiller number

\begin{tabular}{|c|c|c|c|}
\hline \multirow[t]{3}{*}{ Treatment } & \multicolumn{3}{|c|}{ Plant age (DAT = days after treatment) } \\
\hline & 3 & 24 & 31 \\
\hline & \multicolumn{3}{|l|}{ Plant height } \\
\hline $\mathrm{V}_{1} \mathrm{~T}_{0}$ & $19.88 \pm 0.12 b c$ & $27.50 \pm 2.46 \mathrm{~b}$ & $24.60 \pm 0.44 b$ \\
\hline $\mathrm{V}_{1} \mathrm{~T}_{1}$ & $16.58 \pm 1.66 \mathrm{a}$ & $25.53 \pm 1.50 \mathrm{~b}$ & $23.67 \pm 1.23 b$ \\
\hline$V_{1} T_{2}$ & $18.15 \pm 2.24 a b c$ & $25.67 \pm 2.01 \mathrm{~b}$ & $22.62 \pm 2.76 \mathrm{~b}$ \\
\hline $\mathrm{V}_{1} \mathrm{~T}_{3}$ & $14.52 \pm 1.08 \mathrm{a}$ & $18.17 \pm 2.10 \mathrm{a}$ & $18.17 \pm 1.59 \mathrm{a}$ \\
\hline $\mathrm{V}_{2} \mathrm{~T}_{0}$ & $16.50 \pm 0.66 \mathrm{a}$ & $26.70 \pm 3.45 b$ & $27.42 \pm 3.39 c$ \\
\hline$V_{2} T_{1}$ & $18.33 \pm 3.17 \mathrm{abc}$ & $26.67 \pm 4.69 \mathrm{~b}$ & $26.42 \pm 3.99 c$ \\
\hline$V_{2} T_{2}$ & $21.33 \pm 0.52 \mathrm{c}$ & $26.00 \pm 1.09 b$ & $26.92 \pm 2.18 \mathrm{c}$ \\
\hline $\mathrm{V}_{2} \mathrm{~T}_{3}$ & $17.88 \pm 3.59 a b c$ & $26.22 \pm 1.36 \mathrm{~b}$ & $28.50 \pm 0.75 \mathrm{~d}$ \\
\hline $\mathrm{LSD}_{0.05}$ & 3.57 & 4.57 & 3.72 \\
\hline \multirow[t]{2}{*}{ Treatment } & \multicolumn{3}{|l|}{ Tiller number } \\
\hline & 3 & 10 & 17 \\
\hline $\mathrm{V}_{1} \mathrm{~T}_{0}$ & $2.83 \pm 2.29 \mathrm{ab}$ & $3.33 \pm 1.04 \mathrm{ab}$ & $3.83 \pm 1.44 a b$ \\
\hline$V_{1} T_{1}$ & $2.50 \pm 0.87 \mathrm{a}$ & $3.00 \pm 1.32 \mathrm{a}$ & $3.33 \pm 1.04 \mathrm{a}$ \\
\hline$V_{1} T_{2}$ & $4.00 \pm 0.87 \mathrm{~d}$ & $4.00 \pm 0.87 \mathrm{c}$ & $4.17 \pm 1.04 \mathrm{bcd}$ \\
\hline$V_{1} T_{3}$ & $3.33 \pm 0.58 \mathrm{~cd}$ & $3.50 \pm 0.50 \mathrm{~b}$ & $4.67 \pm 0.58 d$ \\
\hline$V_{2} T_{0}$ & $5.00 \pm 0.50 \mathrm{e}$ & $5.50 \pm 0.50 \mathrm{~d}$ & $5.83 \pm 0.29 \mathrm{e}$ \\
\hline$V_{2} T_{1}$ & $3.83 \pm 0.29 \mathrm{~d}$ & $4.00 \pm 0.00 \mathrm{c}$ & $5.17 \pm 1.15 d$ \\
\hline$V_{2} T_{2}$ & $3.67 \pm 0.29 \mathrm{~d}$ & $4.00 \pm 0.50 \mathrm{c}$ & $4.17 \pm 0.58 \mathrm{bcd}$ \\
\hline$V_{2} T_{3}$ & $3.17 \pm 0.29 \mathrm{bc}$ & $3.50 \pm 0.50 \mathrm{~b}$ & $4.00 \pm 0.87 \mathrm{bc}$ \\
\hline $\mathrm{LSD}_{0.05}$ & 0.37 & 0.48 & 0.63 \\
\hline
\end{tabular}

The Numbers followed by same letter in the same row means insignificant difference.

Table 5 - The effect of soil water table on yield component of two shallot varieties

\begin{tabular}{llllll}
\hline \multirow{2}{*}{ No. } & Parameter & \multicolumn{2}{l}{ F-value } & Coefficient of variance (\%) \\
\cline { 3 - 5 } & & $\mathrm{V}$ & $\mathrm{T}$ & $\mathrm{VT}$ & \\
\hline 1. & Fresh weight of harvested plant (shoot + bulb) & $4.07^{\mathrm{ns}}$ & 5.24 & $0.08^{\mathrm{ns}}$ & 42.84 \\
2. & Bulb fresh weight & $5.48^{*}$ & $3.92^{*}$ & $0.10^{\text {ns }}$ & 47.32 \\
3. & Bulb dry weight & $46.42^{*}$ & $1.65^{\text {ns }}$ & $0.62^{\text {ns }}$ & 51.92 \\
4. & Bulb number & $0.73^{\text {ns }}$ & $0.79^{\text {ns }}$ & $0.66^{\text {ns }}$ & 29.40 \\
5. & Bulb diameter & $9.54^{*}$ & $1.37^{\text {ns }}$ & $0.16^{\text {ns }}$ & 33.72 \\
6. & Harvest Index & $9.13^{*}$ & $1.73^{\text {ns }}$ & $1.24^{\text {ns }}$ & 10.68 \\
\hline & F Tabel at 5\% & 4.60 & 3.34 & 3.34 & \\
& F Tabel at 1\% & 8.86 & 5.56 & 5.56 & \\
\hline
\end{tabular}

Note: $n s=$ not significantly different; ${ }^{*}=$ significantly different at $p<0.05$; and ${ }^{* *}=$ significantly different at $p<0.01$.

The difference on shallot variety gave significant effect on yield component since planting for 11 days. Total plant (shoot + bulb) fresh weight of Pancasona was higher than Tajuk in all water table treatments. The lowest weight was obtained from $20 \mathrm{~cm}$ of water table below soil surface treatment. Control treatment (without water table treatment) of two varieties had higher weight compared to other treatments with $18.17 \mathrm{~g}$ and $21.83 \mathrm{~g}$ for Pancasona and Tajuk variety (Figure 1).

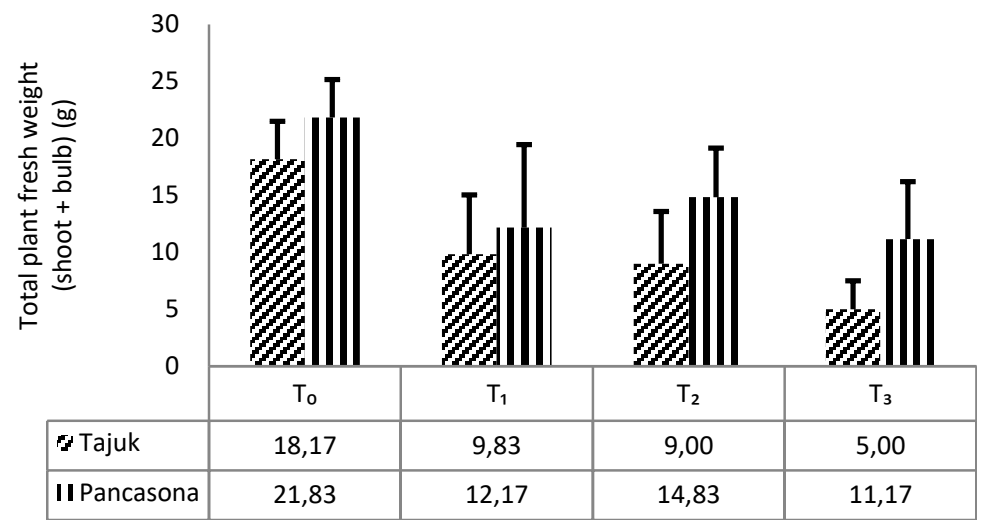

Figure $1-$ Total plant fresh weight (shoot + bulb) of two shallot varieties 


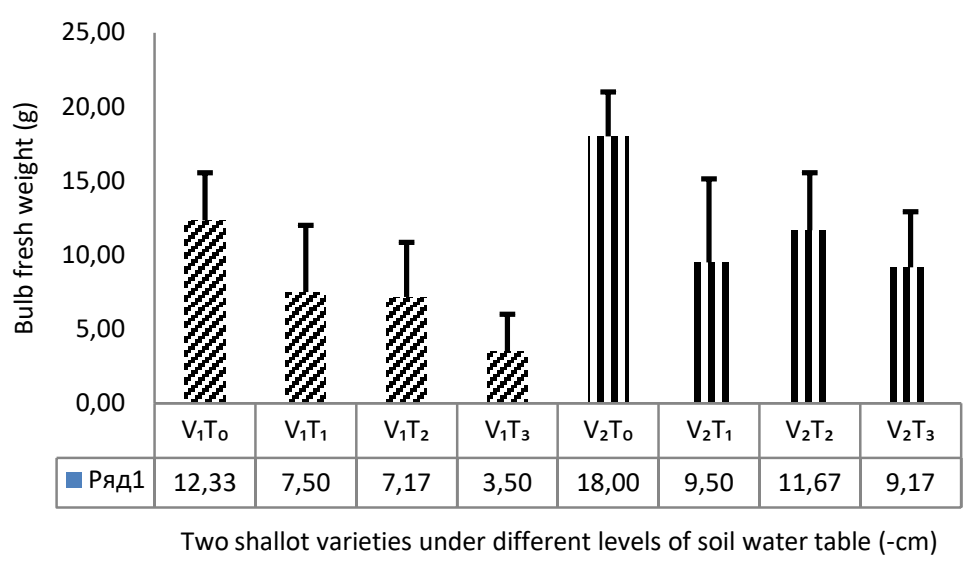

Figure 2 - Bulb fresh weight of two shallot varieties under different levels of soil water table

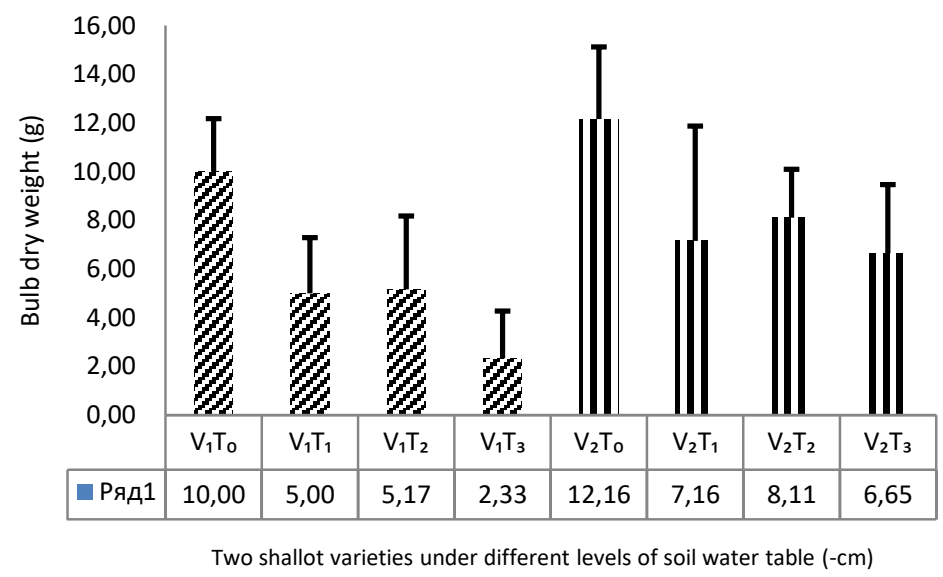

Figure 3 - Bulb dry weight of two shallot varieties under different levels of soil water table

Similar to total plant fresh weight, bulb fresh and dry weight both had the same pattern where the combination treatments of Tajuk showed a lower result compared to Pancasona. The highest weights from both varieties were obtained from control (without water table treatment) with $12.33 \mathrm{~g}$ and $18.00 \mathrm{~g}$ of bulb fresh weight and $10.00 \mathrm{~g}$ and $12.66 \mathrm{of}$ dry weight for Tajuk and Pancasona, respectively (Figure 2 and 3 ).

\section{DISCUSSION OF RESULTS}

Based on the research, it was resulted that two observed shallot varieties still could tolerate water table condition until $20 \mathrm{~cm}$ below soil surface as seen from their growth for 11 days started from planting (Table 1). Table 2 also indicates that there was no significant difference of growth parameters under different levels of soil water table. Along with this result, Susila et al. (2013) reported that Bima Brebes shallot variety still could increase its yield even with $25 \mathrm{~cm}$ of water table below soil surface.

Tajuk variety generally had lower growth ability compared to Pancasona as seen in all growth parameters. Based on variety description, Tajuk is shallot variety introduced from Thailand that suited to be cultivated in lowland area in Nganjuk Regency. While Pancasona is a shallot variety bred by Vegetable Research Center that can well adapt in lowland area with 6-85 $\mathrm{m}$ above sea level. A relatively high ability of growth component was plant height which was only effective at the end of growth phase compared to leave number affected by 24 DAT to 31 DAT, moreover tiller number affected since 0 DAT to 31 DAT.

Generally, results showed that shallot could grow and have better yield under normal water condition (control treatment) as can be seen in vegetative phase shown by Table 2-3 where the plant height, leaf number, and tiller number had some decreases under soil water 
table treatment especially in $20 \mathrm{~cm}$ of water table below soil surface. This showed that the water table until $20 \mathrm{~cm}$ below soil surface did not support shallot growth. Based on a research by Susilawati et al. (2019) about the use of biochar at different levels of soil water table towards shallot, there was a contradictive result where shallot still could grow well at the water depth until $20 \mathrm{~cm}$ below soil surface when provided with biochar treatment. This was assumed that the addition of biochar had improved water binding capacity of the planting media, so there was adequate water supply for the plant. Dou et al. (2014) stated that the addition of biochar affected soil physical characteristics which could increase water absorption capacity thus could directly affect wider root development zone resulted in the plant's amenity to get water and nutrition need for its growth. The other research result showed that the use of biochar sized less than $1 \mathrm{~mm}$ had better effect than biochar sized 1 or more than $1 \mathrm{~mm}$ on rice growth (Kartika et al., 2018; Kartika et al., 2019). The condition of 20 $\mathrm{cm}$ water level below media surface had better probability for the root to grow and develop as there was soil pore filled with oxygen due to the addition of biochar. A research result by Lakitan et al. (2018) stated that bean's root could not survive in a water saturated condition.

In accordance with the vegetative phase, yield components result had rather similar pattern in which the highest total plant weight (shoot and bulb) and bulb fresh weight and bulb dry weight was obtained in Pancasona variety compared to Tajuk variety. Likewise, at the treatment of water table, the yield was decreasing with the depth until $20 \mathrm{~cm}$ below soil surface (Figure 1-3). However, this condition is also contradictive when biochar was added to the planting media. This was due to biochar originated from oil palm empty fruit bunch was composed by $25.6 \%$ of C-organic and 19.4 of $\mathrm{C} / \mathrm{N}$ ratio which could improve soil physical characteristics (Santi dan Goenadi, 2012). This condition resulted in better plant growth and development for both shoots and bulbs. The use of biochar was considered would be very helpful in improving soil under less optimum conditions as in this research where water availability was limited. Another research mentioned that there was a huge biomass increase if the biochar was given to the soil without fertilizer (infertile soil) compared to the soil with fertilizer (Haefele et al. 2011; Carter et al. 2013).

\section{CONCLUSION}

Based on the results, it was concluded that Pancasona variety had better growth compared to Tajuk variety under several levels of soil water table. Soil water table of $20 \mathrm{~cm}$ below soil surface could decrease the growth and yield of shallot for both Pancasona and Tajuk variety.

\section{ACKNOWLEDGEMENTS}

Thank you to Rector of Universitas Sriwijaya and LP2M (Lembaga Penelitian dan Pengabdian pada Masyarakat) Universitas Sriwijaya that had facilitated and funded this research through research contract no. 0149.64/UN9/SB3.LP2M.PT/2019 on June 27, 2019.

\section{REFERENCES}

1. Agricultural Data and Information Center. 2016. Shallot Outlook. Ministry of Agriculture.

2. Bella, S.E and R.Padrikal. 2018. The use of biochar from oilpalm empty fruit bunch as NPK fertilizer substitute to increase agricultural land quality. Journal of Applied Agricultural Science and Technology 2(1):27-34.

3. Carter S, Shackley S, Sohi S, Suy TB, Haefele S (2013). The impact of biochar application on soil properties and plant growth of pot grown lettuce (Lactuca sativa) and cabbage (Brassica chinensis). Agronomy. 3(2): 404-418.

4. Dou L, Komatsuzaki M, Nakagawa M. 2012. Effects of biochar, mokusakueki and bokashi application on soil nutrients, yields and qualities of sweet potato. J. Agriculture Science and Soil Science. 2: 318-327. 
5. Glaser, B., J. Lehmann and W. Zech. 2002. Ameliorating Physical and Chemical Properties of Highly Weathered Soils in The Tropics with Charcoal -A review. Biology and Fertility of Soils. 35: 219-230.

6. Haefele et al, 2011. Effects and fate of biochar from rice residues in rice-based systems. Field Crops Res. 121:430-441.

7. Jackson, M.B. et al, 2015. Plant response and adaptation to flooding and submergence stress. Short Communication. Special Issue: Plant Responses to Low Oxygen Environments Introduction to the Special Issue: Electrons, water and rice fields. 1-10.

8. Kartika et al, 2018. Effects of particle size and application rate of rice-husk biochar on chemical properties of tropical wetland soil, rice growth and yield.AJCS 12(05):817 826 . doi:10.21475/ajcs.18.12.05.PNE 1043.

9. Kartika. 2019. Penggunaan oryza glaberrima dan biochar untuk meningkatkan produktivitas padi di lahan rawa lebak Sumatera Selatan. Disertasi (Tidak Dipublikasikan). Program Pasca Sarjana Universitas Sriwijaya.

10. Kurnianingsih, A., Susilawati, dan M. Sefrila. 2018a. Karakter Pertumbuhan Tanaman Bawang Merah Pada Berbagai Komposisi Media Tanam. J.Hort.Indonesia Volume 9(3):167-173. Terakreditasi No: 2/E/KPT/2015.

11. Kurnianingsih, A., Susilawati dan R. Hayatullah. 2018b. Respon Pertumbuhan dan Hasil Tanaman Bawang Merah (Allium Cepa L.) Varietas Bima pada berbagai Komposisi Media Tanam . Prosiding Seminar Nasional Lahan Suboptimal 2018, Palembang 18-19 Oktober 2018 ISBN: 978-979-587-801-8 120.

12. Malik AI, Ailewe TI, Erskine W (2015) Tolerance of three grain legume species to transient waterlogging. AoB Plants.7. Plv040. doi:10.1093/aobpla/plv040.

13. Meihana, Lakitan B, Harun MU, Susilawati. 2019a. Optimalisasi produkstivitas Iahan rawa lebak melalui aplikasi amelioran dan penanaman sayuran yang adaptif. Dissertation (Unpublished) Program Pasca Sarjana Universitas Sriwijaya.

14. Meihana et al, 2019b. Steady shallow water table did not decrease leaf expansion rate, specific leaf weight, and specific leaf water content in tomato plants. AJCS 11(12):16351641.doi: 10.21475/ajcs.17.11.12.pne808.

15. Rajagukguk, R. 2019. Sumsel dan Kalsel Paling Siap Dioptimalkan Lahan Rawanya. https://www.inews.id/finance/makro/sumsel-dan-kalsel-paling-siap-dioptimalkan-lahanrawanya.

16. Santi L.P. and D.H. Goenadi. 2012. The use of biochar from oilpalm empty fruit bunch as microbes carrier for aggregate stabilization. Buana Sains Vol.12 (1): 7-14.

17. Santi LP. 2017. The use of biochar from oilpalm empty fruit bunch to increase nutrient absorption and carbon sequester on lithic hapludults soil media in oilpalm nursery. Jurnal Tanah dan Iklim. 41(1): 9-16.

18. South Sumatra Statistic Center. 2018. Vegetable production in South Sumatra Province.

19. Statistic Indonesia, 2017. Statistics of Indonesian seasonal vegetable and fruit crops. Statistic Indonesia. $111 \mathrm{pp}$.

20. Sudarsono, 2018. Solok Jadi Produsen Bawang Merah di Sumatera. https://ekbis.sindonews.com/read/1327710/34/solok-jadi-produsen-bawang-merah-disumatera-1533384154. [Accessed on March 13, 2019].

21. Susilawati., Muhammad Ammar dan Mu'arif. 2018a. The effect of planting media composition on growth and yield of shallot (Allium cepa L.). Proceeding of National Seminar on Suboptimal Land.18-19 Oktober 2018, Palembang.

22. Susilawati., Ammar Muhammad., Kurnianingsih Astuti., Irmawati., Adhistia Yoanna dan Yuniar Fajri. 2018b. The Correlation between Growth Characters and Yield of Shallot (Allium ascalonicum L.) due to the Application of Nitrogen, Phosphate and Potassium Fertilizers. RJOAS. Vol. 9(81): 502-509.

23. Susilawati and Lakitan B. 2019. Cultivation of Common bean (Phaseolus vulgaris L.) Subjected to Shallow Water Table at Riparian Wetland in South Sumatra, Indonesia. Australian Journal of Crop Science. 13(10): 98-104.

24. Susilawati et al, 2019. The use of biochar and soil water table at one month after planting on growth and yield of shallot. Jurnal Suboptimal Vol.8 (2). 